\title{
High rate X-ray imaging using multi-GEM detectors with a novel readout design
}

\author{
S. Bachmann ${ }^{\mathrm{a}}$, S. Kappler ${ }^{\mathrm{ab} *}$, B. Ketzer ${ }^{\mathrm{a}}$, Th. Müller ${ }^{\mathrm{b}}$, L. Ropelewski ${ }^{\mathrm{a}}$, F. Sauli $^{\mathrm{a}}$, and E. Schulte ${ }^{\mathrm{c}}$ \\ ${ }^{a}$ CERN, EP division, CH-1211 Geneva 23, Switzerland \\ bInstitut für Experimentelle Kernphysik, Karlsruhe University, Germany \\ ${ }^{\mathrm{c}}$ Institute of Physics, Helsinki University, Finland
}

Modern micropattern gas counters are able to detect soft X-rays with high spatial resolution. Based on the principle of gas amplification, this detector type has a wide dynamic range and provides energy-selection during image reconstruction. One of the most recent developments is the Multi-GEM, an efficient, high-rate capable micropattern gas detector. Owing to the separation of gas amplification and readout stage, this detector allows high flexibility in the geometry of the readout structure.

With a two-dimensional projective readout, GEM detectors have shown good capability in providing X-ray absorption radiographies of small mammals. We present a detector with a novel design where the readout board consists of micropads connected to three layers of strips in such a way that two-dimensional spatial reconstruction and an unambiguous resolution of events with multiple hits is possible.

Keywords: X-ray imaging; Gas Electron Multiplier; GEM; high rate radiation detector; micropad readout; multiple hits; unambiguous resolution

PACs: $29.40 . \mathrm{Cs} ; 77.22 . \mathrm{Jp} ; 51.70 .+\mathrm{f}$

\section{Introduction}

Initially developed for High Energy Physics, gas detectors are used in a wide range of applications. Recent developments showed that modern micropattern gas counters are able to detect soft X-rays with high spatial resolution [1-3]. A review of the application of gas detectors in radiology can be found in [4].

Exploiting the gas amplification of this detector type provides wide dynamic range and, if operated in a single photon counting mode, energyselection during image reconstruction.

The Gas Electron Multiplier (GEM) [5], which was initially invented as a preamplification stage for micropattern gas detectors, is a two-side copper-clad Kapton foil, perforated with a high density of holes. These holes are etched using a photolithographic process with a pitch of usually $140 \mu \mathrm{m}$ and diameters of about $70 \mu \mathrm{m}$ (so-called

\footnotetext{
${ }^{*}$ Corresponding author: Steffen.Kappler@cern.ch
}

standard geometry). Application of a potential difference between upper and lower electrode creates a high electric field inside the holes. Inserted in the drift gap of a gas detector, nearly all drifting electrons are guided into these holes where they undergo proportional gas amplification.

Stacking several GEMs in a cascade leads to the multi-GEM detector (fig. 1). This efficient, high-rate capable detector is one of the most recent developments in the family of micropattern gas detectors. The effective gain obtained with a GEM detector can be adapted to the needs of the application choosing the number of GEMs and the voltage across each one. The big advantage of this detector type is the separation of gas amplification and readout stage, resulting in the fact, that the readout signal is only due to motion and collection of the electrons. This separation provides not only a margin of safety in case of discharges occurring in the GEM foils, it also allows high flexibility in the geometry of the 


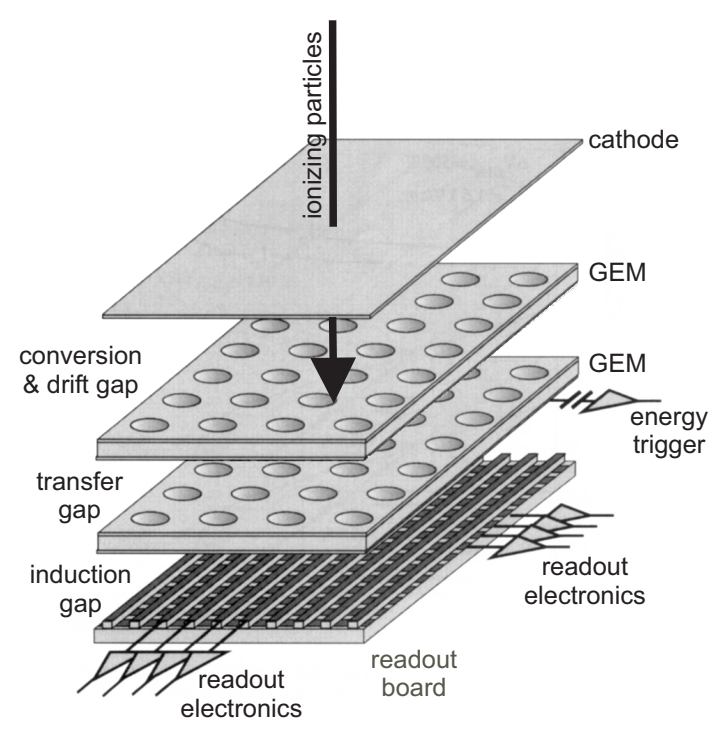

Figure 1. Schematic view of a multi-GEM detector.

readout structure. Experience from High Energy Physics showed, that in a single GEM operated in Ar: $\mathrm{CO}_{2}$ (70:30) effective gains well above $10^{3}$ can be reached. The rate capability of these detectors was verified in a set of studies up to fluxes exceeding $10^{5} \mathrm{~Hz} / \mathrm{mm}^{2}$. Using double-GEM detectors for X-ray detection, discharge probability and aging-effects can be neglected [6]. With good resolution in time and space and the possibility to read the lower electrode of the lower GEM to trigger the readout electronics, the multi-GEM detector meets the basic requirements for fast Xray imaging devices.

\section{A double-GEM detector for X-ray ab- sorption radiography}

A double-GEM detector with an active area of $10 \times 10 \mathrm{~cm}^{2}$ was built to provide X-ray absorption radiographies of small mammals. The two-dimensional projective microstrip readout of this detector (fig. 2) was equipped with electronics based on the PreMux128 chip [7]. This charge

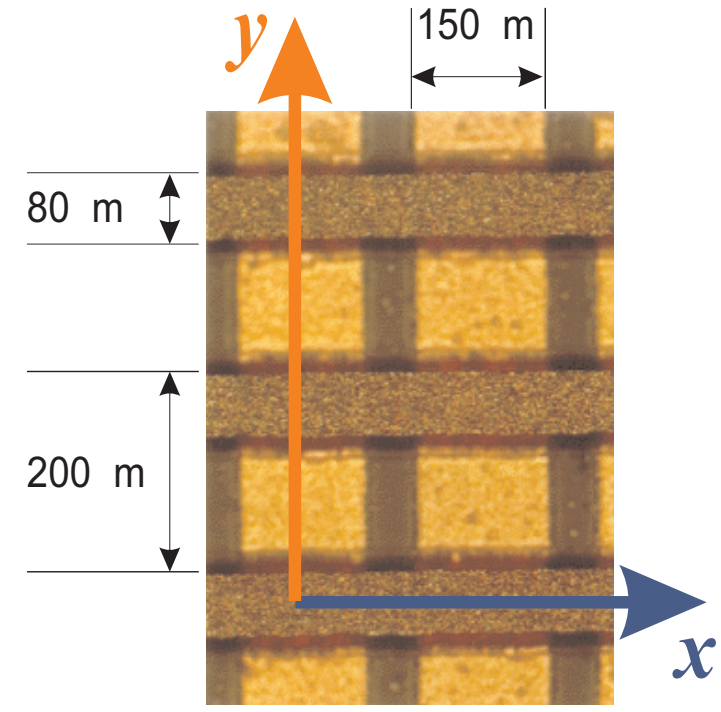

Figure 2. Microscope photograph of the twodimensional projective microstrip readout.

sensitive electronics, developed for High Energy Physics applications, is not optimized for imaging purposes.

The detector was operated with a gas mixture of $\mathrm{Ar}: \mathrm{CO}_{2}$ (70:30) at atmospheric pressure. The sensitive gap between drift cathode and first GEM was chosen to be $3 \mathrm{~mm}$, the transfer gap between the GEMs and the induction gap to the readout board was $2 \mathrm{~mm}$. Fields of $3.3 \mathrm{kV} / \mathrm{cm}$ in the drift gap, $4.0 \mathrm{kV} / \mathrm{cm}$ in the transfer gap, and $4.3 \mathrm{kV} / \mathrm{cm}$ in the induction gap were selected. The GEMs (standard geometry) were operated at a voltage difference of $430 \mathrm{~V}$ each.

Figure 2 shows a photograph of the microstrip readout structure used. It consists of two layers of strips, each at a pitch of $200 \mu \mathrm{m}$, and separated by $25 \mu \mathrm{m}$ Kapton. The strip width is $80 \mu \mathrm{m}$ in the upper, and $150 \mu \mathrm{m}$ in the lower layer, resulting in a well correlated charge sharing, essential for an efficient detection on both coordinates [8].

With an X-ray tube providing a beam of $8 \mathrm{keV}$ photons, this detector was used to generate e.g. the X-ray absorption radiography of a bat, shown 

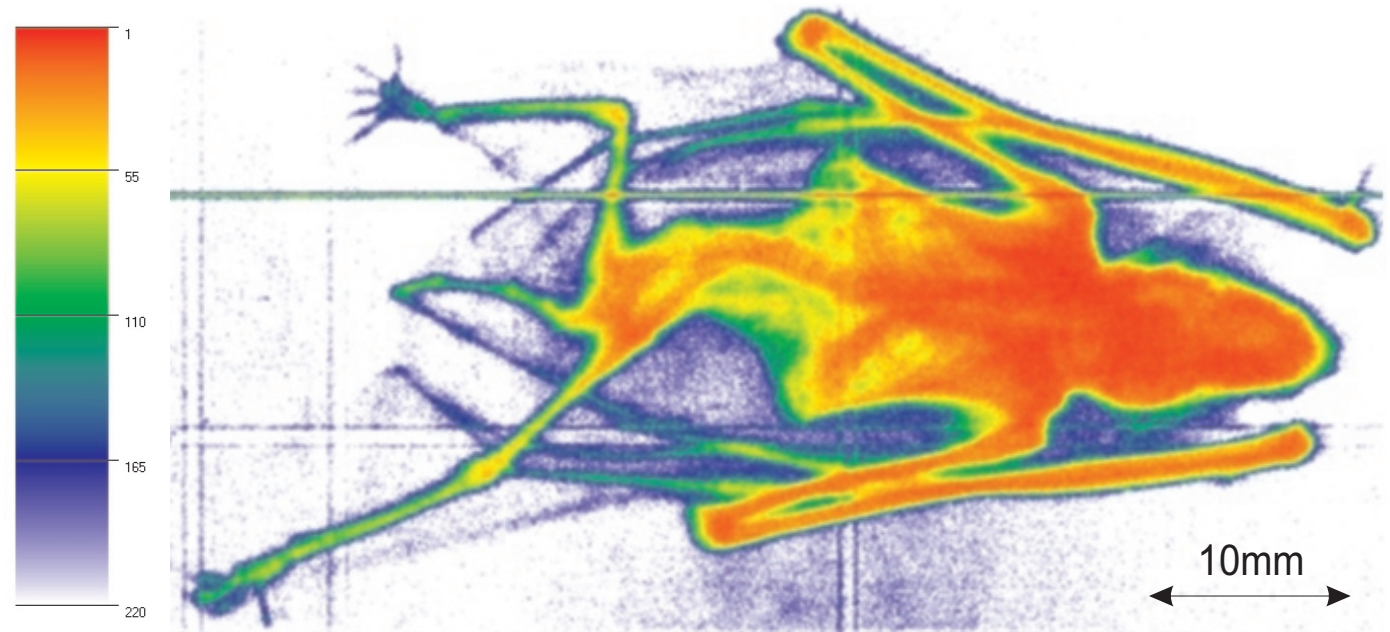

Figure 3. X-ray absorption image of a small mammal. The color scale indicates the number of photons counted in the pixels.

in figure 3. This $60 \times 35 \mathrm{~mm}^{2}$ image with pixel sizes of $100 \times 100 \mu \mathrm{m}^{2}$ consists of 36.5 million photon counts. In this image, the skeleton including spine, vertebrae, and even the small ribs can be recognized.

Despite the good imaging properties of this readout structure, at higher rates (exceeding a few hundred $\mathrm{kHz}$ over the detector area) the reconstruction efficiency is limited by ambiguities due to multiple hits within one readout cycle, resulting from the intrinsic time resolution of the readout electronics (around hundred ns). With a conventional two-dimensional projective readout structure, no information is obtained which would allow the resolution of those multiple hits. (We remind the reader that for monoenergetic Xrays the correlation in charge normally can not be used.)

\section{Resolution of multiple hits - a novel readout design}

In order to obtain the missing information, a third projection can be added. Therefore, a novel readout board was designed, consisting of micropads, which are alternately connected to three different layers of strips, crossing at an angle of $120^{\circ}$ (fig. 4). The strips are embedded in layers of insulating photo-polymer and have a pitch of $520 \mu \mathrm{m}$. The uppermost layer contains the micropads, which have an outer diameter of $300 \mu \mathrm{m}$. By choosing the coordinate system indicated in figure 4 (with the axes $u, v$, and $w$ perpendicular to the corresponding strips) each point fulfills the following plane equation:

$u+v+w=0$

For clusters covering three projections, this equation can be used for the resolution of events with multiple hits. An example for a three-hit event is shown in figure 5 . There, the charge correlation helps to couple the three coordinates of one hit, but for the remaining two hits a different procedure has to be chosen. One possible approach is the following: In a run with $N$ hits, an algorithm for cluster recognition provides the coordinates $u_{1} \ldots u_{N}, v_{1} \ldots v_{N}$, and $w_{1} \ldots w_{N}$. A combinatorial check,

$\left|u_{i}+v_{j}+w_{k}\right|<\delta$

which is derived from equation (1) and includes possible avalanche dispersions (represented by 


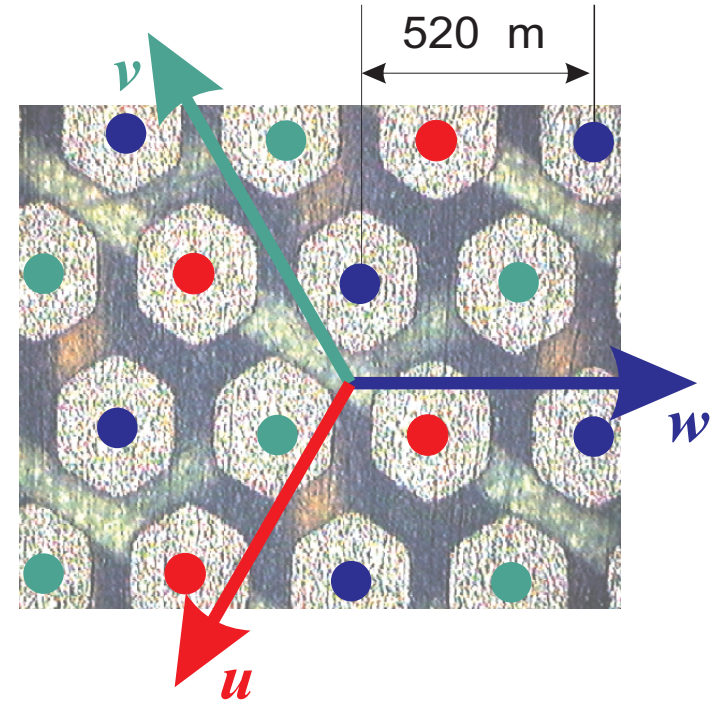

Figure 4. Microscope photograph of the novel micropad readout. The micropads are alternately connected to three different layers of strips.

$\delta)$, gives the valid combinations $\left(u_{i}, v_{j}, w_{k}\right)$. To record the resolved hits in an image with Cartesian coordinates $(x, y)$, a coordinate transformation is necessary.

The present prototype of this readout board was produced at CERN by R. de Oliveira and consists of seven layers of conductive patterns, embedded in an insulator and carried by a fibreglass plate. The production process consists of the following steps: The first set of readout strips (coordinate $u$ ) is chemically etched into a copper layer on the support plate. In the second step, a photo-polymer layer is laminated onto the strips. Covered by a photolithographic mask, it is exposed to UV light. The uncovered regions polymerize and the remaining material is removed afterwards. Then, the resulting holes in the insulator are filled with a conductive silverepoxy, and after hardening the surface is polished. The following layers (coordinates $v$ and $w$, pads, and connectors) are added the same way, starting from the second step.

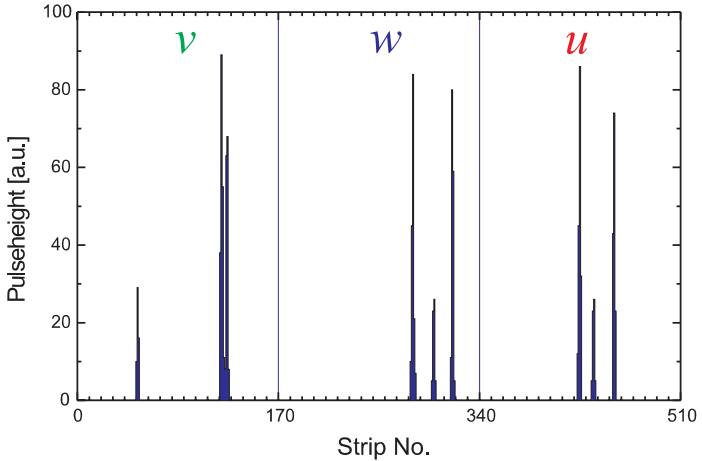

Figure 5. Typical event with three hits over the detector area.

To check properties and imaging performance of this readout circuit, it was integrated into a double-GEM detector with a geometry similar to the one described above. Connected to the same electronics and operated in the conditions mentioned, an energy resolution of $\Delta E_{\mathrm{fwhm}} / E=$ $20.1 \%$ could be achieved, and the charge sharing was well correlated with ratios of $1: 1$ ( $v$ to $w$ ) and $1: 1.2$ ( $u$ to $v$ and $u$ to $w$ ), as shown in figure 6.

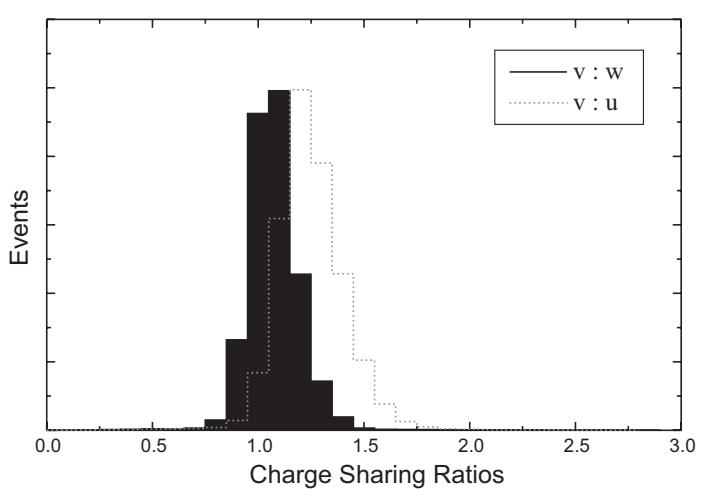

Figure 6. Charge sharing ratios $v: w$ and $v: u$. 

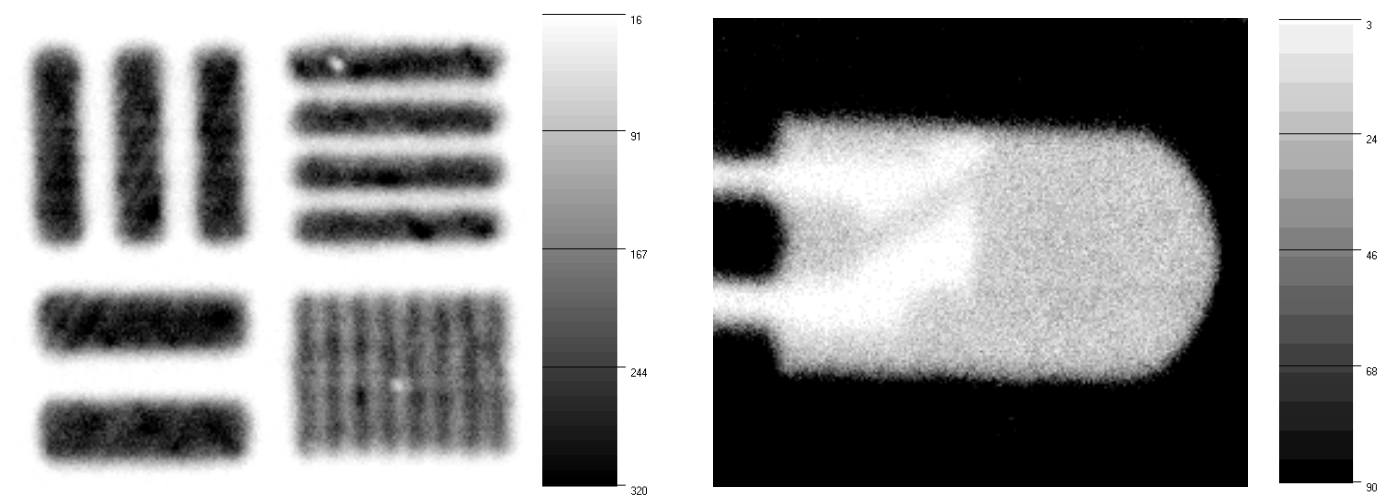

Figure 7. X-ray absorption images of slit mask and LED, taken with the micropad readout structure. The grey scales indicate the number of photons counted in the pixels.

With the $8 \mathrm{keV} \mathrm{X}$-rays, the radiography of a slit mask and a LED were taken (fig. 7). These $10 \times 10 \mathrm{~mm}^{2}$ images with pixel sizes of $50 \times 50 \mu \mathrm{m}^{2}$ demonstrate the imaging performance of this readout structure. Even the small slits of $300 \mu \mathrm{m}$ width, which are $210 \mu \mathrm{m}$ apart, can be separated.

The multi-hit capability is mainly limited by clusters overlapping in one projection. In $\mathrm{Ar}: \mathrm{CO}_{2}$ (70:30, $1 \mathrm{~atm})$, a mean cluster size of $770 \mu \mathrm{m}$ (fwhm) was measured, and thus the probability of overlapping clusters is not negligible. To quantify the multi-hit capability, a set of $10^{5}$ single-hit events was recorded at low rate. In order to simulate pure $N$-hit runs, $N$ record sets of this run were superimposed. Afterwards, an algorithm with a relatively primitive cluster recognition was used to resolve the $N$ hits according to the reconstruction principle mentioned above. For an active area of $10 \times 10 \mathrm{~mm}^{2}$, this algorithm was able to find $66 \%$ of the hits in a 2-hit run, and $50 \%$ in a 3-hit run. Optimizing the detector for smaller cluster sizes and developing better reconstruction algorithms, would even improve these numbers.

\section{Conclusions}

The studies carried out showed that multiGEM detectors are able to detect soft X-rays at high fluxes with good spatial resolution. The novel readout design provides sufficient resolution and reconstruction of multi-hit events, and the present prototype with the hexagonal pad design shows reasonable imaging qualities.

High rate $\mathrm{X}$-ray imaging, Čerenkov ring imaging, and the readout of Time Projection Chambers offer promising perspectives for the application of projective pad readout designs.

\section{REFERENCES}

1. E.A. Babichev et al., NIM A310 (1991) 449454.

2. H.J. Besch, NIM A360 (1995) 277-282.

3. M. Dixit et al., IEEE Trans. 47 (1998) 809.

4. A. Sharma, Gaseous Micropattern Detectors In Astrophysics, Radiology, And Plasma Physics, CERN-OPEN/2000-313, October 31, 2000.

5. F. Sauli, NIM A386 (1997) 531-534.

6. S. Bachmann et al., Progress in development of GEM detectors, Proceedings of the Vienna Conference on Instrumentation, February 19$24,2001$.

7. L.L. Jones, PreMux128 specification, version 2.3, RD20 internal document, January 1995.

8. S. Bachmann et al., Performance of GEM detectors in high intensity particle beams, CERN-EP/2000-116, August 2000. 